\title{
Molecular contrast optical coherence tomography: a pump-probe scheme using indocyanine green as a contrast agent
}

\author{
Zahid Yaqoob \\ Emily McDowell \\ Jigang $\mathrm{Wu}$ \\ Xin Heng \\ Jeff Fingler \\ Changhuei Yang \\ California Institute of Technology \\ Department of Electrical Engineering \\ Biophotonics Laboratory \\ 4 Moore Building, M/C 136-93 \\ 1200 E. California Blvd. \\ Pasadena, California 91125
}

\begin{abstract}
The use of indocyanine green (ICG), a U.S. Food and Drug Administration approved dye, in a pump-probe scheme for molecular contrast optical coherence tomography (MCOCT) is proposed and demonstrated for the first time. In the proposed pump-probe scheme, an optical coherence tomography (OCT) scan of the sample containing ICG is first acquired. High fluence illumination $\left(\sim 190 \mathrm{~kJ} / \mathrm{cm}^{2}\right)$ is then used to permanently photobleach the ICG molecules_-resulting in a permanent alteration of the overall absorption of the ICG. A second OCT scan is next acquired. The difference of the two OCT scans is used to determine the depth resolved distribution of ICG within a sample. To characterize the extent of photobleaching in different ICG solutions, we determine the cumulative probability of photobleaching, $\phi_{B, \text { cum }}$, defined as the ratio of the total photobleached ICG molecules to the total photons absorbed by the ground state molecules. An empirical study of ICG photobleaching dynamics shows that $\phi_{B, \text { cum }}$ decreases with fluence as well as with increasing dye concentration. The quantity $\phi_{B, \text { cum }}$ is useful for estimating the extent of photobleaching in an ICG sample (MCOCT contrast) for a given fluence of the pump illumination. The paper also demonstrates ICG-based MCOCT imaging in tissue phantoms as well as within stage 54 Xenopus laevis. () 2006 Society of Photo-Optical Instrumentation Engineers. [DOI: $10.1117 / 1.2360525]$
\end{abstract}

Keywords: optical coherence tomography; molecular contrast; indocyanine green; medical and biological imaging; pump-probe.

Paper 05314RR received Oct. 17, 2005; revised manuscript received May 13, 2006; accepted for publication Jun. 20, 2006; published online Oct. 26, 2006.

\section{Introduction}

Optical coherence tomography ${ }^{1}$ (OCT) is an important noninvasive biomedical tool for high resolution ${ }^{2}$ imaging of biological samples to a depth of a few millimeters. Since its inception, OCT has matured into a useful diagnostic tool for ophthalmic applications. ${ }^{3-6}$ OCT's applications for imaging other areas of the human body include endoscopic imaging of the gastrointestinal (GI) $\operatorname{tract}^{7}$ and bladder ${ }^{8}$ for tumor detection, monitoring and risk assessment of vulnerable lipid plaques in the vascular system, ${ }^{9}$ monitoring intracoronary stenting, ${ }^{10}$ and tracking structural weakness associated with tooth decay. ${ }^{11}$ While basic OCT imaging is able to render depth resolved structural images of the target, more sophisticated OCT imaging strategies can provide additional functional information such as flow (through Doppler OCT),${ }^{12}$ tissue structural arrangement (through birefringence OCT), ${ }^{13}$ and depth resolved spectral signatures (through spectral OCT) ${ }^{14,15}$ In recent years, a new and exciting functional OCT method, known as molecular contrast optical coherence to-

Address all correspondence to Zahid Yaqoob, California Institute of Technology, Biophotonics Laboratory, Department of Electrical Engineering, 4 Moore Building, M/C 136-93, 1200 E. California Blvd., Pasadena, California 91125, Tel. (626) 395-4711; Fax (626) 395-8952; E-mail: zyaqoob@caltech.edu mography (MCOCT) ${ }^{16-24}$ has been introduced that combines the major advantages of fluorescence microscopy (chemical or biological contrast agent's detection and imaging capability) and OCT (higher spatial resolution and depth penetration).

In this paper, we explore the use of indocyanine green (ICG), which is used in medical diagnostics ${ }^{25}$ and photodynamic therapy, ${ }^{26}$ in a MCOCT scheme based on the photobleaching mechanism of the dye. This work differs in its contrast mechanism from an earlier work with ICG in a MCOCT scheme, where the contrast agent's distribution inside the sample was determined from agent's spectral differential absorption. ${ }^{21}$

Aqueous solutions of ICG degrade over time; the process is accelerated in the presence of light with spectral band that is coincident with the ICG optical absorption peak. This degradation is attributable to the photobleaching of the ICG. In addition to the ground and first excited states, ICG has three states of interest, namely the triplet $(T), P$-isomer $(P)$, and bleached $(B)$ states, in which the molecules can reside (see Fig. 1). An excited molecule will generally relax directly back into the ground state, but it does have a small probability of

1083-3668/2006/11(5)/054017/12/\$22.00 @ 2006 SPIE 
Yaqoob et al.: Molecular contrast optical coherence tomography...

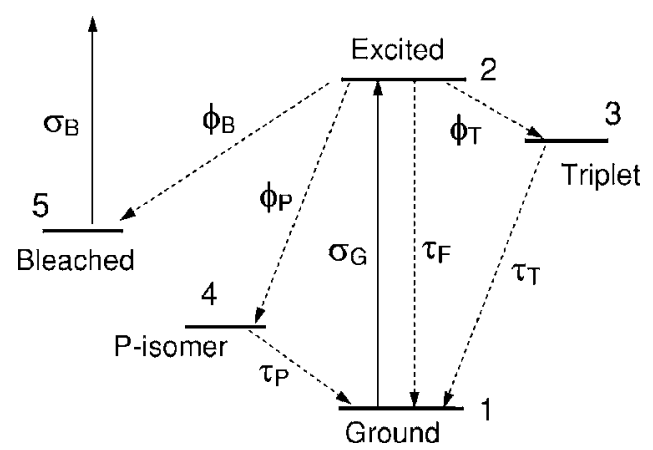

Fig. 1 Schematic of electronic state diagram of ICG. $\sigma_{G}$ is the ground state absorption cross section, whereas $\phi_{T}, \phi_{P}$, and $\phi_{B}$ are quantum yields of triplet $(T), P$-isomer $(P)$, and bleached $(B)$ states.

transiting into any of these states. The quantum yields $\phi_{T}, \phi_{P}$, and $\phi_{B}$ of the three states are very low $\left(\sim 10^{-5}\right.$ to $\left.10^{-6}\right) .{ }^{27}$ The pump-probe MCOCT scheme makes use of the fact that molecules that are temporarily shelved (for the case of triplet and $P$-isomer states) or permanently bleached (for the case of bleached state) have a different absorption spectrum from ground state molecules to elicit contrast. We note that relatively short lifetimes associated with the triplet and $P$-isomer states (triplet state lifetime, $\tau_{T} \sim 700 \mu$ s and $P$-isomer state lifetime, $\left.\tau_{P} \sim 10 \mathrm{~ms}\right)^{27}$ will require elaborate pump-probe schemes for contrast data acquisition. In comparison, the permanency of the photobleached state is appealing in that given enough time, all the molecules in an illuminated sample of ICG solution can be transitioned into the bleached state; there is no stringent pump intensity requirements or fast signal acquisition scheme requirements in this case.

The implementation scheme based on the use of photobleaching can be summarized as follows. A baseline OCT scan of the sample containing a contrast agent is first acquired, followed by photoillumination of the sample. The photoillumination excites the molecules and repeatedly cycles the molecules through the ground-excited state transition. The molecules have a small chance of ending up in the photobleached state during each transition; as such, given a high enough fluence, the majority of the molecules will eventually end up in the photobleached state. A second OCT scan is then acquired. The two OCT scans, which are different as the absorption spectrum of the contrast agent has changed, are thus processed to determine the distribution of the contrast agent within the sample. This paper provides an understanding of the ICG dynamics in the proposed pump-probe scheme and demonstrates the MCOCT technique in tissue phantoms as well as in an animal model.

Since the proposed pump-probe scheme uses the photobleached state, it is important to understand and quantify the photobleaching mechanism in ICG. Most notable among previous works on the photodegradation process in ICG is the work of Holzer et al. ${ }^{28}$ The study, however, is based on either very low (e.g., $10 \mu \mathrm{M}$ ) or very high (e.g., $1.5 \mathrm{mM}$-toxic level ${ }^{29}$ ) concentrations of ICG. Moreover, the work was predominantly geared toward characterizing the stability of above two concentrations of ICG (in different solvents) based on the initial probability of photobleaching. In this work, we have studied moderate (e.g., $\sim 10$ to $\sim 140 \mu \mathrm{M}$ ) concentra- tions of ICG. From this study, we can characterize the variation of the initial probability of photobleaching changes with different ICG concentration and the dependency of the probability of photobleaching on light fluence. We will like to note that it is the cumulative probability of photobleaching that is needed to estimate the time required to photobleach a given ICG sample with a specified irradiance. Therefore, the initial probabilities (such as the ones reported for 10 or $1.5 \mathrm{mM}$ ICG concentrations in Ref. 28) cannot be used to accurately estimate the photobleaching extent of 200 and $400 \mu \mathrm{M}$ ICG concentrations used in the demonstration of proposed pumpprobe-based MCOCT scheme.

This paper is divided into six sections. In Sec. 2, we will report on our experimental characterizations of the photobleaching mechanism. In Sec. 3, we will elaborate on the computation scheme by which we will extract MCOCT information from our experimental data. In Sec. 4, we will describe the MCOCT experiment and report on our experimental findings. In Sec. 5, we will discuss the adaptation of this MCOCT scheme for clinical applications. Finally, we will conclude with a summary of our findings.

\section{Photobleaching Dynamics of ICG}

As an initial step in the study of the photobleaching dynamics of ICG, we performed a simple experiment to verify the stability of the photobleached state. Glass cuvettes (from Starna Cells, Inc.) with $1 \mathrm{~cm}$ path length were filled with $50 \mu \mathrm{M}$ ICG (SDB8662, H. W. Sands Corp.) solutions in $1 \%$ bovine serum albumin (BSA) and water, and exposed to $790 \mathrm{~nm}$ illumination (incident optical power $\sim 1.2 \mathrm{~W}$ ) for several hours. Figures 2(a) to 2(c) show absorption cross-section spectra as well as photographs of both unbleached and bleached samples of $50 \mu \mathrm{M}$ ICG in BSA and water. Notice that the unbleached samples of ICG solution in BSA and water are both green in color. However, the bleached samples of ICG solution in BSA and water possess shades of orange and gray, respectively. The bleached samples of ICG in BSA and water were also observed over several days to see if any recovery of the dye from its bleached state had occurred. For this purpose, the bleached samples were kept in the dark and two additional sets of absorption spectra were acquired-one on the third day, and the other on the fifth day [see Figs. 2(d) and 2(e)]. A quantitative comparison of the measured absorption spectra of bleached ICG samples shows no signs of recovery from the bleached state, which confirms that the photobleached state is permanent. The permanence of ICG photobleaching illustrates the usefulness of bleached state transition in a pump-probe scheme for OCT - the focus of this study. We note that the extent of change in absorption cross section $\Delta \sigma=\sigma_{G}-\sigma_{B}$, where $\sigma_{G}$ and $\sigma_{B}$ are the ground state and bleached state absorption cross sections, respectively, is a useful parameter to estimate achievable contrast from a given ICG sample. From Fig. 2(a), it can be seen that ICG solution in BSA shows a higher contrast change compared to that in water. This observation, along with the fact that ICG shows high affinity to bind with proteins, ${ }^{27}$ indicates that higher contrast should be expected from tissue locales where ICG is attached to proteins provided the ICG samples are completely bleached. 


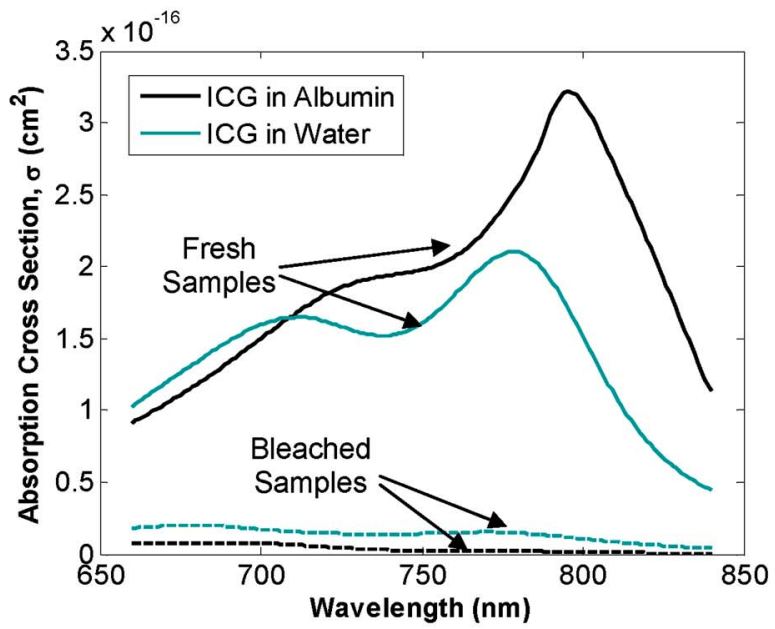

(a)

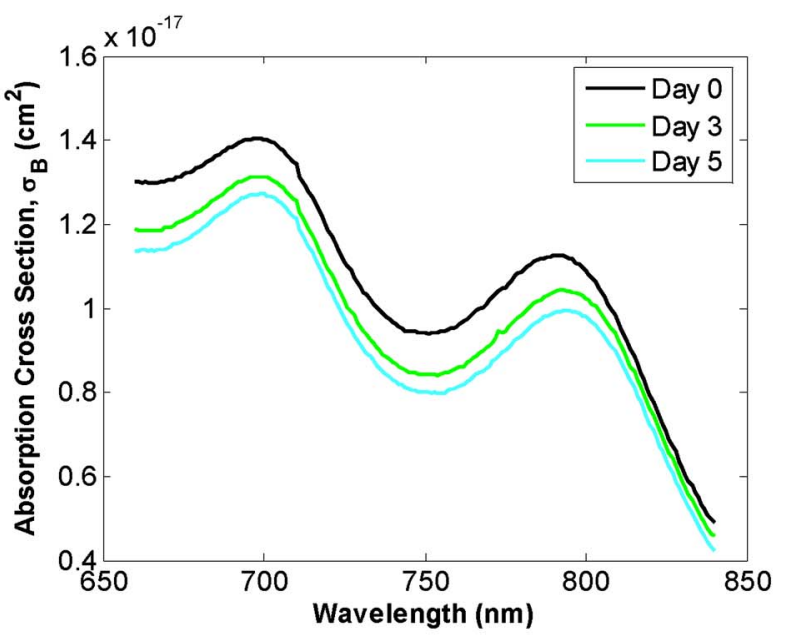

(d)
ICG in 1\% BSA

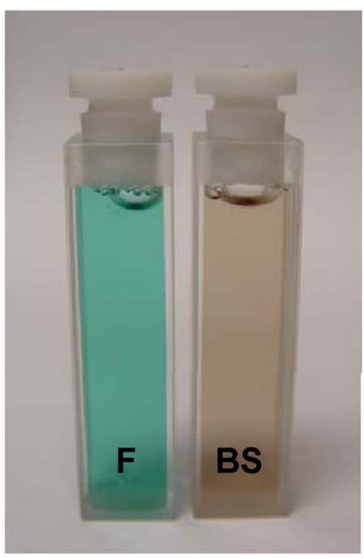

(b)
ICG in $\mathrm{H}_{2} \mathrm{O}$

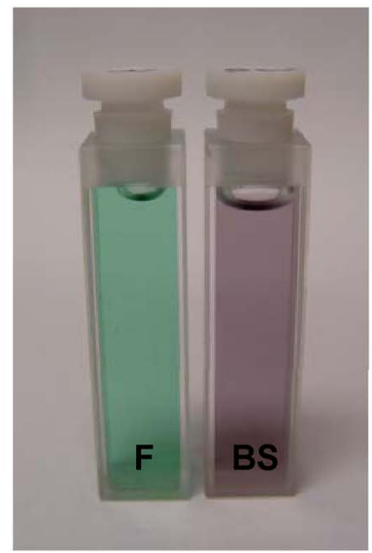

(c)

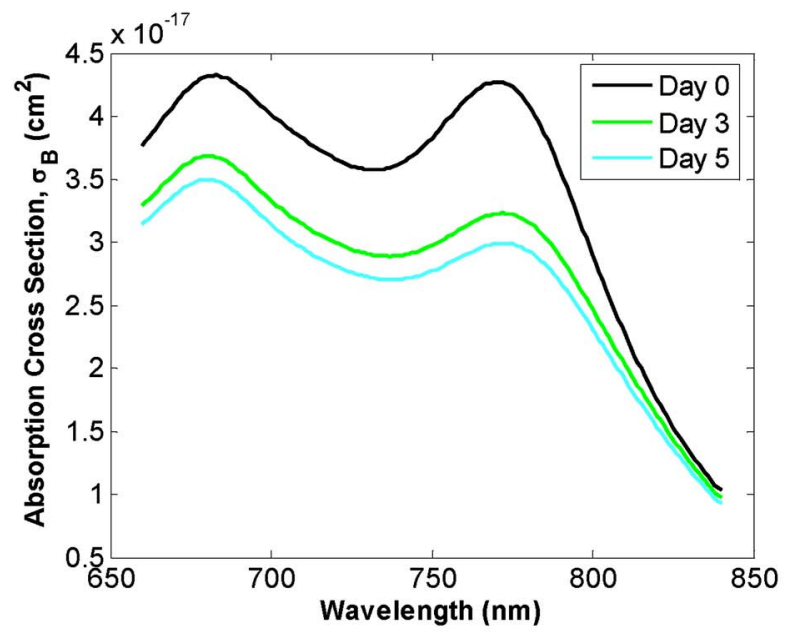

(e)

Fig. 2 Shows (a) absorption cross-section spectra and (b) and (c) pictures of unbleached as well as bleached samples of ICG in 1\% BSA and water. ICG concentration is $50 \mu \mathrm{M}$ in all the cases. (d) and (e) show absorption spectra of bleached samples of ICG in BSA and water, respectively, acquired at different times. F: fresh; BS: bleached sample.

Figure 3 shows the setup to characterize the degradation dynamics of ICG. A fraction of the collimated beam from a Ti:sapphire laser (Spectra Physics Tsunami, Model 3941$\mathrm{X} 3 \mathrm{BB}$ ) is redirected using a beamsplitter (BS) to form the reference beam. The major portion of the light (signal beam) passes straight through a square cuvette with $\sim 2 \mathrm{~mm}$ diameter circular hole. Glass cover slips are used on each side to hold the ICG samples. The path length $L$ of the specially designed circular cross-section cuvette is $1.52 \mathrm{~mm}$ (see Fig. 3 ). Notice that the diameter of the incident collimated laser beam is approximately the size of the circular hole to reduce effects due to diffusion. A balanced photodetector (New Focus, Inc.) is used to measure the transmission through the ICG samples over time. Since, (a) ICG shows high affinity to bind with proteins (which would be the case if injected into a protein environment) and (b) agarose was used (to reduce the effects due to diffusion) in the proof-of-concept demonstrations, $50 \mu \mathrm{M}$ ICG solutions were prepared in deionized (DI) water, $1 \% \mathrm{BSA}$, and $0.8 \%$ agarose for initial study. ICG so- lutions in BSA and agarose solutions were also prepared using DI water. Transmission time traces were acquired for different irradiance levels ranging from $25 \mathrm{~W} / \mathrm{cm}^{2}$ to $\sim 80 \mathrm{~W} / \mathrm{cm}^{2}$. Before each transmission measurement, the system was balanced (or calibrated) by using the corresponding solvent (water, agarose, or BSA) in an identical cuvette and adjusting the free-space attenuators $A_{1}$ and $A_{2}$ accordingly. Note that fresh samples of ICG were used for each transmission measurement. Figures 4(a)-4(c) show the transmission time traces of $50 \mu \mathrm{M}$ ICG in water, $1 \% \mathrm{BSA}$, and $0.8 \%$ agarose, respectively, for different irradiance levels. A visual inspection of the transmission curves in Fig. 4 suggests that ICG solution in BSA shows a higher transmission change when bleached compared to ICG solution in water or agarose. This finding is consistent with the $\Delta \sigma$ values for ICG in water and $1 \%$ BSA, derived from absorption spectra shown in Fig. 2(a).

The absorption of ICG does not change linearly with concentration. This is due to the fact that ICG tends to aggregate in water at higher concentrations; aggregated ICG molecules 


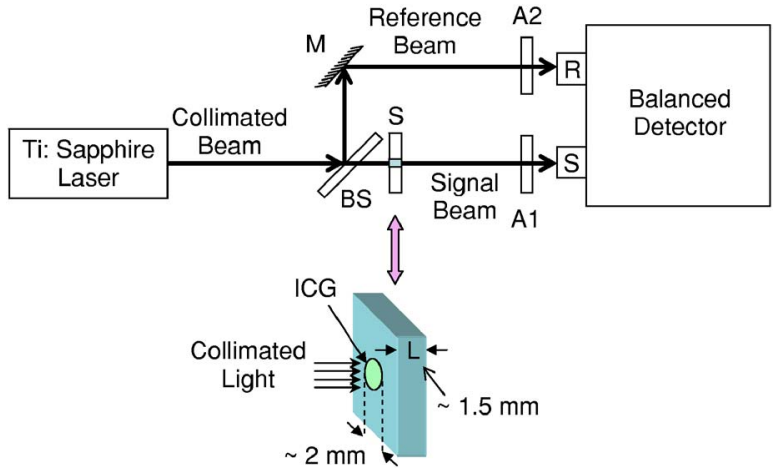

Fig. 3 Experimental setup to study ICG photodegradation dynamics. BS: beamsplitter; M: mirror; Ai: i'th attenuator; R: reference beam; $\mathrm{S}$ : sample beam.

have a different effective absorption cross section. Nevertheless, Beer's law would still be valid if the absorption cross section were known for a given wavelength and ICG concentration $N_{0}$. Assuming that the absorption cross sections of the ground and bleached states do not change during the photobleaching process, the bleached state population density $N_{B}(t)$ can be written as

$$
N_{B}(t)=\frac{1}{\left[\sigma_{G}-\sigma_{B}\right] L} \ln \left[\frac{T(t)}{T_{\min }}\right],
$$

where $L$ is the sample path length and $T(t)$ is the transmittance (the ratio of transmitted light intensity to the input light intensity). $T_{\min }=\exp \left[-\sigma_{G} N_{0} L\right]$ is the minimum transmittance at $t=0$, where $N_{0}$ is the initial concentration of ICG. Because the irradiance decays through the sample, $N_{B}(t)$, in Eq. (1) represents the spatial average of the bleached state population density. Therefore, the probability of photobleaching, defined as the ratio of total number of photobleached molecules to the total number of photons absorbed by ground state molecules, can be mathematically expressed as

$$
\phi_{B, \mathrm{cum}}(t)=\frac{N_{B}(t) \times V}{\int_{0}^{t} \frac{P_{0}}{h \nu}\left[1-T_{G}(\tau)\right] d \tau},
$$

where $V$ is the volume of the ICG sample, $h$ is Planck's constant, $\nu$ is the optical frequency of illumination, $P_{0}$ is the incident optical power, and $T_{G}(t)=T_{\min } \exp \left[\sigma_{G} N_{B} L\right]$ is the transmittance attributed to ground state ICG molecules. It can be seen from Eq. (2) that the probability of photobleaching is a function of time unless the terms in the numerator and denominator vary in a similar fashion in time. Moreover, this approach yields a probability that is cumulative in nature such that at a given time $t, \phi_{B, \text { cum }}(t)$ gives an average probability of photobleaching for time interval $[0, t]$.

The transmission data shown in Fig. 4 were processed to determine the cumulative probability of photobleaching, $\phi_{B, \text { cum }}$, using Eqs. (1) and (2). The cumulative probability of photobleaching was determined for each data set and averaged. Figure 5 shows average $\phi_{B, \text { cum }}$ versus fluence for $50 \mu \mathrm{M}$ ICG in water, $1 \% \mathrm{BSA}$, and $0.8 \%$ agarose; the corresponding initial cumulative probabilities, $\phi_{B, \text { cum,init }}$, were de-
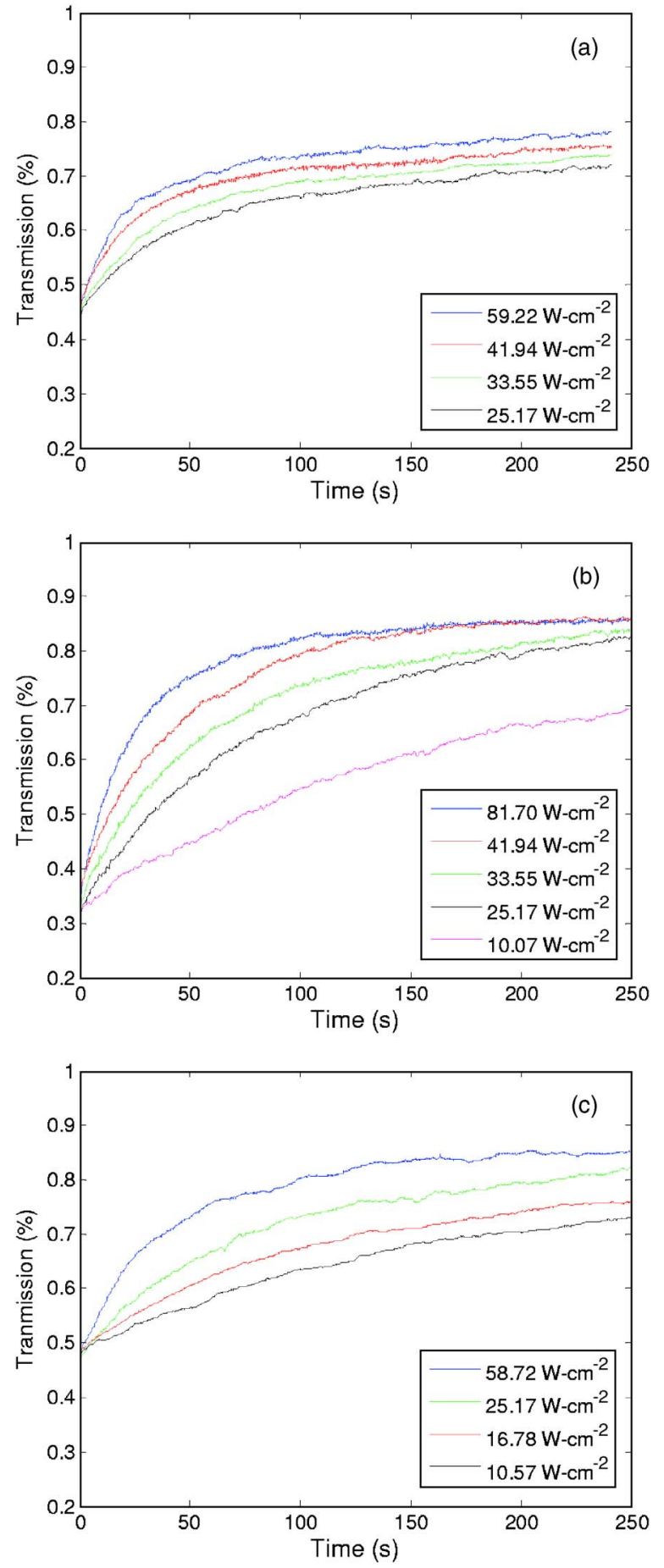

Fig. 4 Transmission curves for $50 \mu \mathrm{M}$ ICG versus time in (a) DI water, (b) $1 \% \mathrm{BSA}$, and (c) $0.8 \%$ agarose for different illumination intensities (irradiance levels).

termined as $(1.2 \pm 0.17) \times 10^{-5}, \quad(0.97 \pm 0.12) \times 10^{-5}$, and $(0.8 \pm 0.3) \times 10^{-5}$, respectively. This empirical study also illustrates that $\phi_{B \text {,cum }}$ decreases with fluence. For instance, in the case of $50 \mu \mathrm{M}$ ICG in water, the probability of photobleaching decreased by $\sim 1 / 3$ of the corresponding

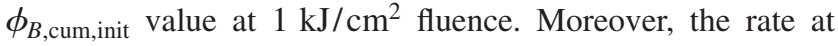
which the cumulative probability of photobleaching decreases decays with increasing fluence levels, indicating that for 


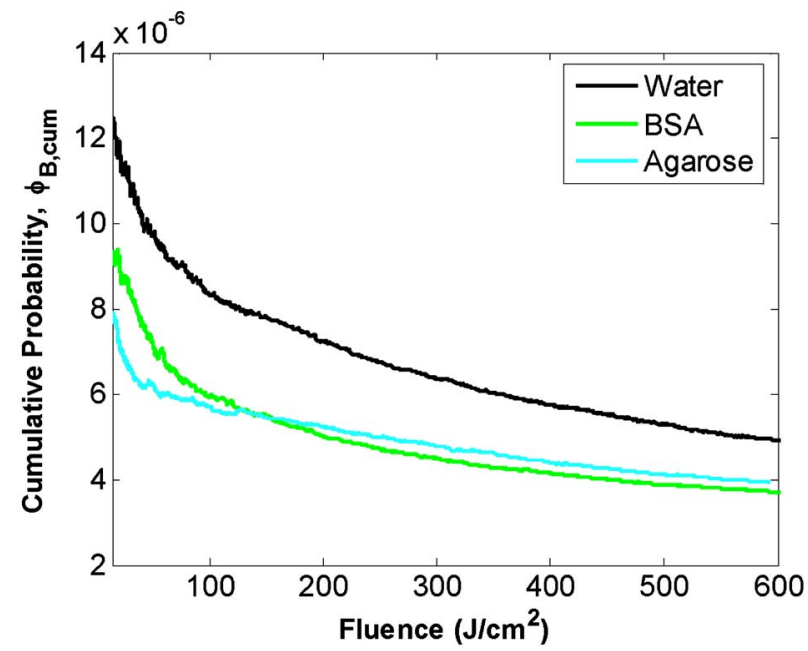

Fig. 5 Cumulative probability $\phi_{B, \text { cum }}$ for $50-\mu \mathrm{M}$ ICG in DI water, $1 \%$ $\mathrm{BSA}$, and $0.8 \%$ agarose. The irradiance in all cases is $33.55 \mathrm{~W} / \mathrm{cm}^{2}$.

higher (e.g., $\geq \sim 10 \mathrm{~kJ} / \mathrm{cm}^{2}$ ) fluence levels, $\phi_{B \text {, cum }}$ will tend toward a final value $\phi_{B \text {,cum,final }}$. We note that the final cumulative probability of photobleaching, $\phi_{B \text {,cum,final, would be }}$ representative of the whole photobleaching event.

We also studied the photobleaching phenomenon as a function of ICG concentrations in DI water. To keep the initial transmissions alike for different concentrations of ICG, cuvettes with different path lengths were assembled. ICG concentrations ranging from 12 to $137 \mu \mathrm{M}$ were used in the study. The irradiance on each sample was $\sim 33 \mathrm{~W} / \mathrm{cm}^{2}$. For each concentration, four sets of measurements were acquired and averaged for better accuracy. The transmission curves were processed to determine cumulative probability of photobleaching versus time. The study shows that although $\phi_{B, \text { cum,init }}$ decreases with increasing ICG concentration $M$ (see

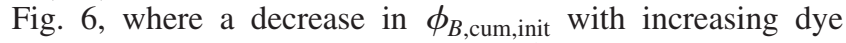
concentration can be clearly seen), the corresponding $\phi_{B, \text { cum,final }}$ values (at $\sim 10 \mathrm{~kJ} / \mathrm{cm}^{2}$ fluence level) converge to $(2.7 \pm 0.6) \times 10^{-6}$. This trend indicates that for high fluence

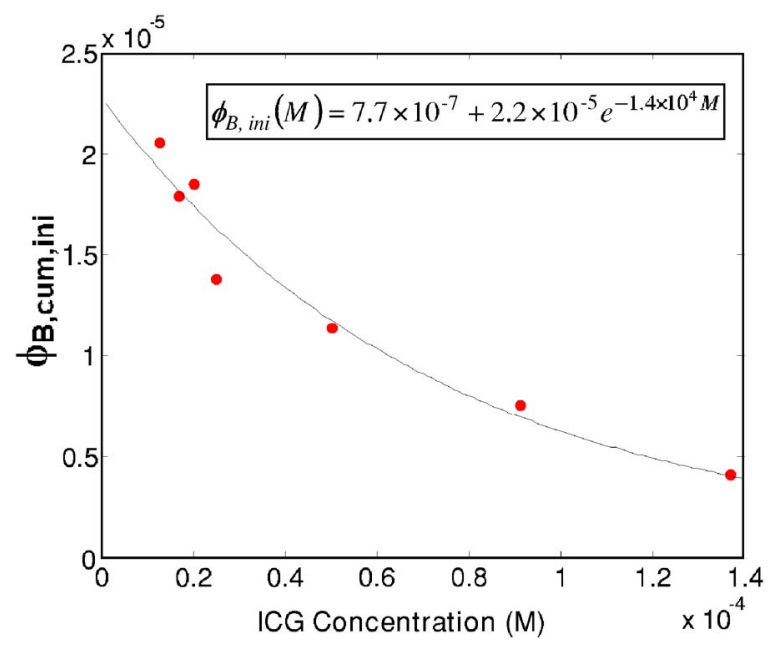

Fig. 6 Initial cumulative probability of photobleaching $\phi_{B, \text { cum, init }}$ versus ICG concentration.

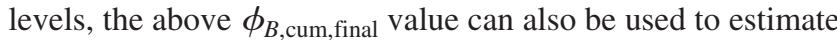
the extent of photobleaching in 200 and $400 \mu \mathrm{M}$ ICG concentrations (used in the MCOCT demonstrations). During this study, we also found the initial probability of photobleaching for $10 \mu \mathrm{M}$ ICG concentration in water to be $1 \times 10^{-5}$, which is approximately two orders of magnitude smaller than that reported in Ref. 28.

To estimate the extent of photobleaching in a given sample of ICG for a specified irradiance $I$, consider a simple model that takes into account only the ground, excited, and bleached states (see Fig. 1). The relevant rate equations can therefore be written as

$$
\begin{gathered}
\frac{\partial N_{E}}{\partial t}=\frac{\sigma_{G} I}{h \nu} N_{G}-\frac{1}{\tau_{E}} N_{E}, \\
\frac{\partial N_{B}}{\partial t}=\frac{\phi_{B, \text { cum }}}{\tau_{E}} N_{E}, \\
\frac{\partial I}{\partial z}=-\sigma_{G} N_{0} I+\left(\sigma_{G}-\sigma_{B}\right) N_{B} I,
\end{gathered}
$$

where, $N_{G}, N_{E}$, and $N_{B}$ are the population densities in the ground, excited, and bleached states, respectively. $I$ is the irradiance, $\tau_{E}$ is the lifetime of excited state, and $h \nu$ is the energy of photons. Since $\partial N_{E} / \partial t \ll N_{E} / \tau_{E}$ and the irradiance $I$ is much less than saturation intensity of the excited state, Eqs. (3a) and (3b) can be combined to yield

$$
\frac{\partial N_{B}}{\partial t}=\phi_{B, \operatorname{cum}} \frac{\sigma_{G} I}{h \nu}\left[N_{0}-N_{B}\right] .
$$

Notice that irradiance $I$ as well as population densities $N_{G}$, $N_{E}$, and $N_{B}$ are functions of depth $z$ as well as time $t$. If $N_{B}(t)$ represents the spatial average of the bleached state population density varying in time, differential equation (3c) can be solved as

$$
I(z)=I_{0} \exp \left(-\left[\sigma_{G} N_{0}+\left(\sigma_{B}-\sigma_{G}\right) N_{B}(t)\right] z\right),
$$

where $I_{0}$ is the illumination intensity at $z=0$. Notice that for small dye concentrations and path length $z$, one can effectively assume $I(z)=I_{0}$. Since the probability of photobleaching $\phi_{B \text {, cum }}$ also changes with time due to its dependency on fluence, we can use $\phi_{B \text {,cum,final }}$ to estimate the extent of photobleaching in a given sample of ICG. A simplified expression for photobleached population density $N_{B}(t)$ can therefore be determined from Eq. (4) as

$$
N_{B}(t)=N_{0}\left(1-\exp \left[\frac{-\phi_{B, \text { cum }, \text { final }} \sigma_{G} I_{0}}{h \nu} t\right]\right) .
$$

Using $\phi_{B \text {,cum,final }}=2.7 \times 10^{-6}$ in Eq. (6), the bleached state population density $N_{B}(t)$ was estimated for 200 and $400 \mu \mathrm{M}$ concentrations of ICG in water. The representative curves shown in Fig. 7 were produced for different irradiances. In each case, the irradiance on the ICG samples was assumed uniform through the sample depth $z$. Notice that in a highly scattering sample, the approximation $I(z)=I_{0}$ will not hold and the above expression will overestimate the extent of pho- 
Yaqoob et al.: Molecular contrast optical coherence tomography...

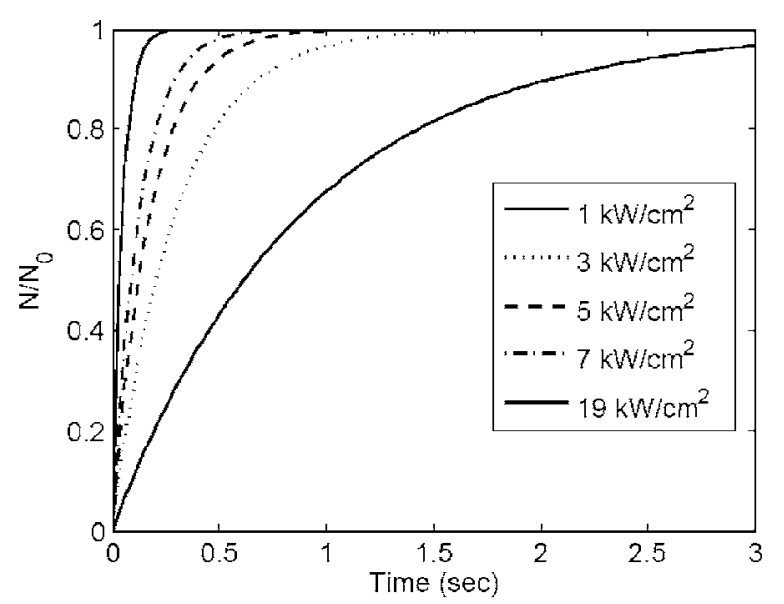

(a)

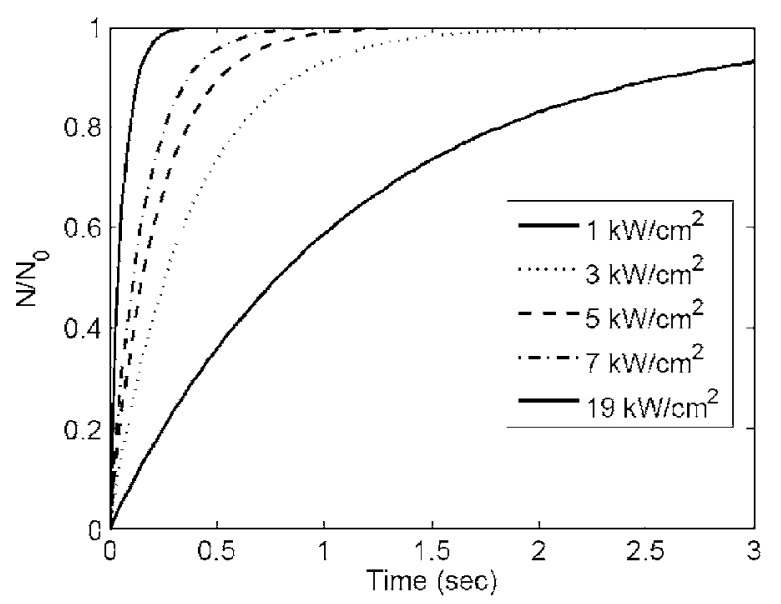

(b)

Fig. 7 Estimated bleached state population density $N_{B}(t)$ for (a) $200 \mu \mathrm{M}$ and (b) $400 \mu \mathrm{M}$ ICG concentrations for different irradiances.

tobleaching process. Nevertheless, the simple exponential model [given by Eq. (6)] will help estimating a lower bound on the time required to photobleach a given sample of ICG. Notice that representative curves in Figs. 7(a) and 7(b) look similar due to the fact that the ground state absorption cross sections for 200 and $400 \mu \mathrm{M}$ ICG concentrations (in water) are comparable. It can also be estimated from Fig. 7 that for the above concentrations of ICG in water, $7 \mathrm{~kW} / \mathrm{cm}^{2}$ irradiance would require approximately $1 \mathrm{~s}$ to completely photobleach 200 and $400 \mu \mathrm{M}$ ICG samples.

\section{Pump-Probe MCOCT Setup}

Figure 8(a) shows our spectral domain MCOCT setup. The experiment uses a high power broadband light source, a galvoscanner-based programmable fiber-optic attenuator (PFOA), and a single $2 \times 2$ fiber-optic coupler. The PFOA is an important element in the design of this pump-probe MCOCT setup as it controls the amount of light reaching the sample during ICG photobleaching as well as OCT signal acquisition. A personal computer both controls the PFOA and acquires data from the spectrometer. Figure 8(b) shows the timing diagram of the pump-probe scheme in Fig. 8(a). From an operational point of view, the PFOA is first set to deliver low optical power, typically less than $100 \mu \mathrm{W}$, so that photobleaching is minimal during the first OCT scan. After the first OCT scan is acquired at time $t_{1}$, the PFOA is set to deliver high power, typically a 1000-fold increase, for a duration $\Delta t$ to photobleach the ICG. The optical power transmitted by the PFOA is then reduced to the same level as for the first OCT scan to acquire the second OCT scan at time $t_{2}$.

At the interferometer, the light collected from the reference and sample arms is spectrally dispersed using a diffraction grating and detected using $N$ different detectors. $N$ can vary from $\sim 100$ to $\sim 1000 \mathrm{~s}$ depending upon the spectrometer employed. For the spectral domain MCOCT setup shown in Fig. 8(a), the spectrally resolved interferometric OCT signal can be mathematically expressed as

$$
\begin{aligned}
P_{\text {interferernce }}(k, z)= & 2 S(k) \eta(k)[1-\eta(k)] \sqrt{R_{R}} \sqrt{R_{s}(\lambda, z)} \\
& \times \exp \left[-\int_{0}^{z}\left[\mu_{a}\left(\lambda, z_{s}^{\prime}\right)+\mu_{s}\left(\lambda, z_{s}^{\prime}\right)\right] d z_{s}^{\prime}\right] \\
& \times \cos \left[2 k\left(z-z_{r}\right)\right]
\end{aligned}
$$

where $k$ is the optical wave number, $S(k)$ is the source spec-

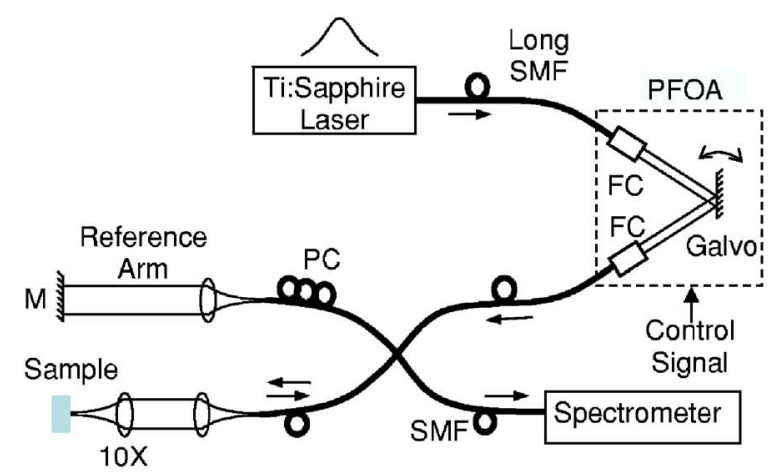

(a)

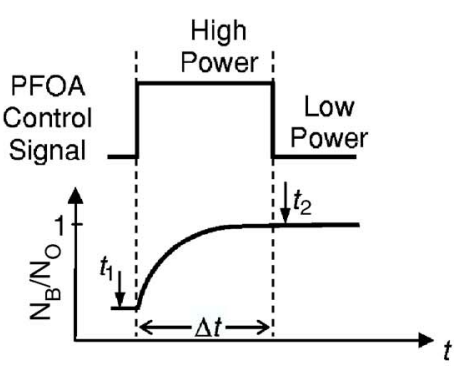

(b)

Fig. 8 (a) Pump-probe MCOCT setup using a high power broadband source such as Ti:sapphire and a galvoscanner-based PFOA for both OCT signal acquisition as well as ICG photobleaching. (b) Timing diagram for the proposed pump-probe scheme. PC; polarization controller; SMF: single mode fiber; PFOA: programmable fiber-optic attenuator; FC: fiber collimator. 
Yaqoob et al.: Molecular contrast optical coherence tomography...

tral profile, $\eta(k)$ is optical power split factor of the $2 \times 2$ coupler (1/2 in an ideal case), $R_{R}$ is the reflectivity of the reference mirror, $R_{s}(\lambda, z)$ is the sample reflectivity at depth $z$, and $\left(z-z_{r}\right)$ is the path length difference between the reference arm mirror and the plane of interest inside the sample. $\mu_{a}(\lambda, z)$ and $\mu_{s}(\lambda, z)$ are the absorption and scattering coefficients, respectively, of the sample at depth $z$. Moreover, the period of the spectral oscillation of the measured signal in $k$ space is proportional to $\left(z-z_{r}\right)$ indicating that an interface with a smaller $\left(z-z_{r}\right)$ value will produce a slower sinusoidal spectral oscillation than an interface with a larger $\left(z-z_{r}\right)$ value. Therefore, a Fourier transform of the spectral measurement will produce a line scan profile (also known as an A-scan) of the sample under study. The additional exponential term accounts for the loss of collected light due to absorption and scattering during the passage into and out of the sample.

In a pump-probe scheme such as the one discussed above using ICG as the contrast agent, the photoillumination permanently alters the absorption coefficient of the dye. In this context, the absorption coefficient can be mathematically written as

$$
\mu_{a}(\lambda, z)=\mu_{a \text {,intrinsic }}(\lambda, z)+\mu_{a, \mathrm{ICG}_{\text {gground }}}(\lambda, z)+\Delta \mu_{a, \mathrm{ICG}}(\lambda, z),
$$

where $\mu_{a \text {,intrinsic }}(\lambda, z)$ is the localized intrinsic absorption coefficient of the sample, $\mu_{a, I_{C G} \text { ground }}(\lambda, z)$ is the localized absorption coefficient component for ICG in the ground state, and $\Delta \mu_{a, \mathrm{ICG}}(\lambda, z)$ is the change in the absorption coefficient between the two OCT scans. The two quantities, $\mu_{a, \mathrm{ICG}_{\text {gground }}}(\lambda, z)$ and $\Delta \mu_{a, \mathrm{ICG}}(\lambda, z)$ can be expressed as

$$
\begin{gathered}
\mu_{a, \text { ICG_ground }}(\lambda, z)=\sigma_{G}(\lambda) N_{0}(z), \\
\Delta \mu_{a, \text { ICG }}(\lambda, z)=\mu_{a, \text { ICG_bleached }}(\lambda, z)-\mu_{a, \text { ICG_ground }}(\lambda, z) \\
=\left[\sigma_{G}(\lambda) N_{G}(z)+\sigma_{B}(\lambda) N_{B}(z)\right]-\sigma_{G}(\lambda) N_{0}(z),
\end{gathered}
$$

where $\sigma_{G}(\lambda)$ and $\sigma_{B}(\lambda)$ are the ground and bleached state absorption cross-section spectra, respectively. $N_{0}(z)$ is the initial ground state population density of ICG at $z$, whereas $N_{G}(z)$ and $N_{B}(z)$ are the ground and bleached state population densities, respectively, when the second OCT scan is acquired. As the irradiance $I$ is much less than saturation intensity of the excited state, $N_{G}(z) \gg N_{E}(z)$, we can safely assume that $N_{0}(z)=N_{G}(z)+N_{B}(z)$. Therefore, Eq. (10) can be simplified as

$$
\Delta \mu_{a, \mathrm{ICG}}(\lambda, z)=\left[\sigma_{B}(\lambda)-\sigma_{G}(\lambda)\right] N_{B}(z)
$$

If $P_{\text {interference_ground }}(\lambda, z)$ and $P_{\text {interference_bleached }}(\lambda, z)$ are the OCT signals acquired before and after the photobleaching of ICG, the change in absorption coefficient is related to the two OCT scans by

$$
\int_{0}^{z} \Delta \mu_{a, \mathrm{ICG}}\left(\lambda, z^{\prime}\right) d z^{\prime}=-\ln \left[\frac{P_{\text {interferernce_bleached }}(k, z)}{P_{\text {interferernce_ground }}(k, z)}\right] .
$$

Solving Eqs. (11) and (12) simultaneously, the distribution of ICG within the sample, represented by $N_{B}(z)$, can be determined as

$$
N_{B}(z)=\frac{d}{d z}\left[\frac{1}{\left\{\sigma_{G}(\lambda)-\sigma_{B}(\lambda)\right\}} \ln \left\{\frac{P_{\text {interferernce_bleached }}(k, z)}{P_{\text {interferernce_ground }}(k, z)}\right\}\right] .
$$

\section{Experiment}

A spectral domain MCOCT system shown in Fig. 8(a) was realized for demonstration. Light from a mode locked Ti:sapphire laser $[\sim 12 \mathrm{~nm}$ full width at half maximum (FWHM) $]$ is coupled into a long $5 / 125 \mu \mathrm{m}$ single mode fiber (SMF) for spectrum broadening. The broadband probe light $(\sim 60 \mathrm{~nm}$ FWHM centered at $800 \mathrm{~nm}$ ) from the SMF is coupled into a $2 \times 2$ single mode fiber-optic coupler via a galvoscannerbased PFOA. Light from the coupler is collimated by lens $L_{1}\left(f_{1}=11 \mathrm{~mm}\right)$. A $10 \times$ microscope objective lens $\left(f_{2}\right.$ $=16.5 \mathrm{~mm}$ ) from Newport Corp. is used to focus the collimated light onto the sample. The calculated $1 / e^{2}$ beam diameter on the sample is $8.2 \mu \mathrm{m}$, which corresponds to a depth of focus of $135 \mu \mathrm{m}$. Light collected from the two arms of the interferometer is detected by a spectrometer designed and built in our laboratory. The spectrometer uses a 1200 lines/mm diffraction grating together with 2048 pixels linear detector array and features $0.07 \mathrm{~nm}$ spectral resolution. The spectral oscillation data acquired by the spectrometer are first rescaled and resampled evenly in $k$ space, before it is Fourier transformed to retrieve the sample depth profile (or A-scan). The rescaled and resampled data are also processed to adjust for any dispersion mismatch between the two arms of the interferometer using the method outlined in Ref. 30. Our laboratory MCOCT system features a measured signalto-noise ratio (SNR) of $98 \mathrm{~dB}$ at 1-ms integration time. The corresponding theoretical shot noise limited SNR is $\sim 108 \mathrm{~dB}$.

In a proof-of-concept demonstration, a $0.84 \mathrm{~mm}$ path length glass cuvette filled with $200 \mu \mathrm{M} \quad\left(N_{0}=12\right.$ $\times 10^{16} \mathrm{~cm}^{-3}$ ) ICG solution in $1 \%$ BSA was used as a sample [see Fig. 9(a)]. The galvoscanner-based PFOA was programmed to deliver $68 \mu \mathrm{W}$ and $10 \mathrm{~mW}\left(\sim 19 \mathrm{~kW} / \mathrm{cm}^{2}\right.$ irradiance based on a beam spot diameter of $8.2 \mu \mathrm{m})$ on the sample for OCT signal acquisition and ICG photobleaching, respectively. The linear detector array integration time for OCT signal acquisition was set to $400 \mu \mathrm{s}$. The ICG was photobleached for duration $\Delta t=2.5 \mathrm{~s}$ between the two OCT scans. Figure 9(b) shows the two A-scans of the $0.84 \mathrm{~mm}$ path length cuvette filled with $200 \mu \mathrm{M}$ ICG solution in $1 \%$ BSA before and after the photobleaching. The first two interfaces of the glass cuvette show no contrast as expected. However, the third and fourth interfaces of the glass cuvette show $\sim 7 \mathrm{~dB}$ of measured contrast, attributable to the change in absorption coefficient of the ICG solution within the cuvette. $\mathrm{Next}$, the $0.84 \mathrm{~mm}$ path length glass cuvette was filled with a 
Yaqoob et al.: Molecular contrast optical coherence tomography...

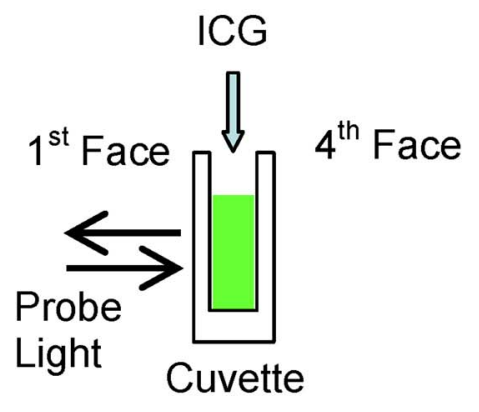

(a)

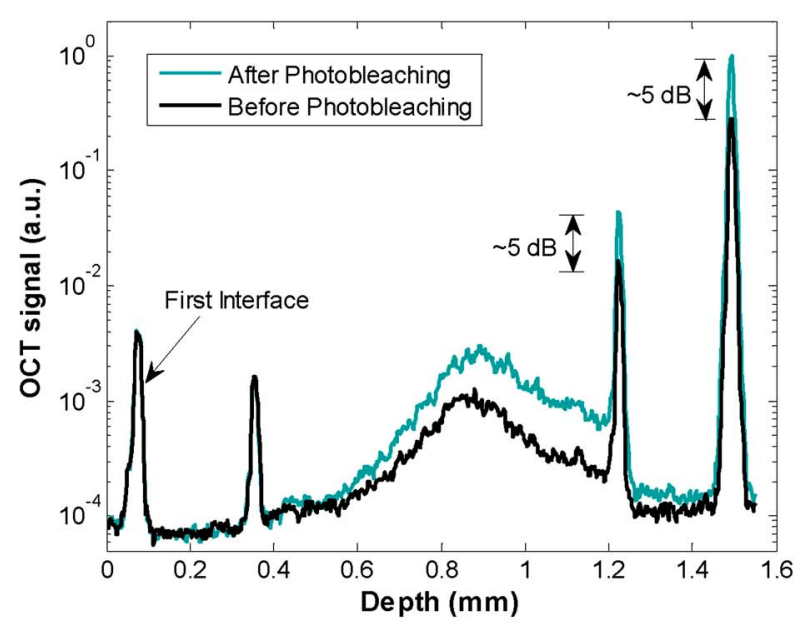

(c)

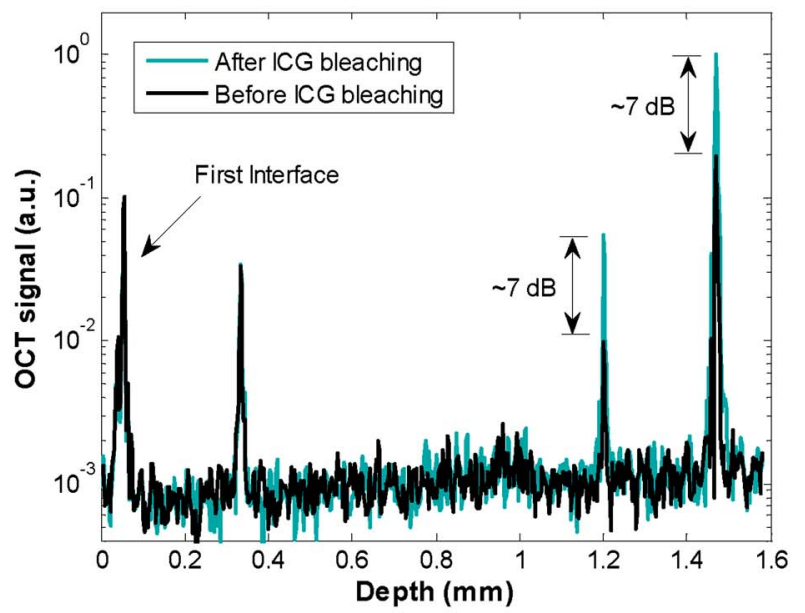

(b)

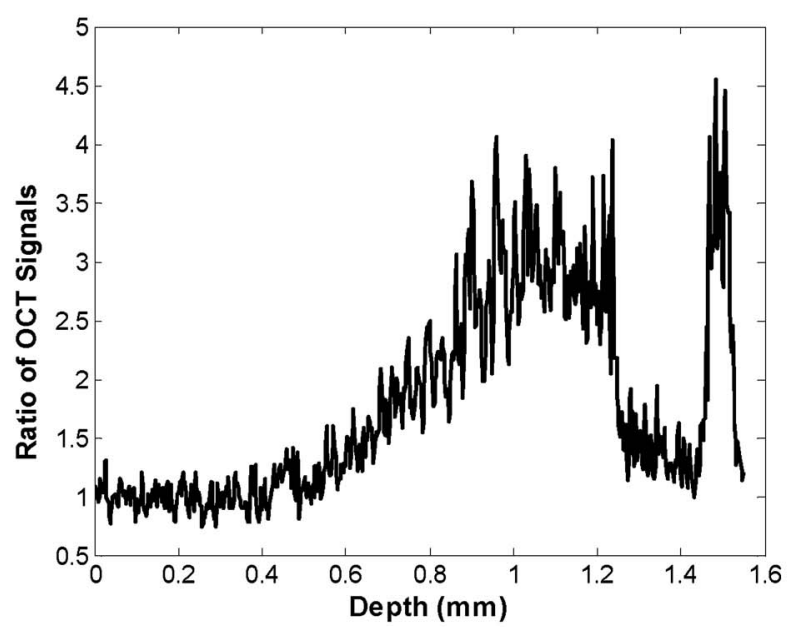

(d)

Fig. 9 (a) Side view of a $0.84 \mathrm{~mm}$ path length cuvette used as a sample. (b) A-scans of the glass cuvette filled with $200 \mu \mathrm{M} \mathrm{ICG}$ solution in $1 \%$ BSA before and after the photobleaching. (c) A-scans of the glass cuvette was filled with a mixture of $200 \mu \mathrm{M}$ ICG and $0.25 \%$ volume concentration $0.1 \mu \mathrm{m}$ latex microspheres before and after the photobleaching. (d) Ratio of the two A-scans is shown in (c).

mixture of $200 \mu \mathrm{M}$ ICG and $0.25 \%(\mathrm{v} / \mathrm{v}) 0.1 \mu \mathrm{m}$ latex microspheres. A series of $10 \mathrm{~A}$-scans was acquired and processed. Figure 9(c) shows averaged A-scans of the glass cuvette before and after the photobleaching. Again, the first two interfaces show no contrast as anticipated. The last two interfaces of the glass cuvette yield a measured contrast of $\sim 5 \mathrm{~dB}$. Notice that the contrast increases with ICG sample depth as it is cumulative in nature [see Eq. (12)]. A ratio of the two averaged A-scans is shown in Fig. 9(d) to support the argument. Using Eq. (13), average bleached state population $N_{B}$ of ICG molecules within the cuvette was determined to be $\sim 9 \times 10^{16} \mathrm{~cm}^{-3}$. We note that at $\sim 19 \mathrm{~kW} / \mathrm{cm}^{2}$ irradiance, it should take less than $0.5 \mathrm{~s}$ to photobleach $>99 \%$ of the ICG molecules (see Fig. 7). However, only 75\% ICG molecules were observed to have transitioned to the photobleached state with an irradiation time of $2.5 \mathrm{~s}$. The reason not all the ICG molecules were bleached can be attributed to the irradiance decay through the highly scattering sample $[0.25 \%(\mathrm{v} / \mathrm{v})$
$0.1 \mu \mathrm{m}$ latex microspheres] and possible diffusion of ICG molecules to and from the region of interest.

Tissue phantoms as well as an animal model were also used in order to evaluate ICG as a contrast agent in the pumpprobe-based MCOCT scheme. In these MCOCT demonstrations, the ICG was used with agarose to reduce the effects due to the diffusion of ICG molecules. In our estimation of pump light dosage for photobleaching of ICG in agarose, it was assumed that $\phi_{B, \text { cum,final }}$ is approximately the same as that for ICG in water. This approximation is fairly reasonable since (a) the initial probabilities of photobleaching for ICG in water and agarose are only different by less than a factor of 2 and (b) $\phi_{B \text {,cum }}$ decreases with fluence in a similar fashion for both the cases (see Fig. 5). Therefore, by approximating $\phi_{B, \text { cum.final }}=2.7 \times 10^{-6}$ in Eq. (6), it is estimated that $19 \mathrm{~kW} / \mathrm{cm}^{2}$ irradiance would require less than $0.5 \mathrm{~s}$ to photobleach $>99 \%$ of the ICG molecules in $400 \mu \mathrm{M}$ ICG sample in agarose. 
Yaqoob et al.: Molecular contrast optical coherence tomography...

The tissue phantoms were prepared by using a glass cuvette comprised of three cells [see Figs. 10(a) and 10(b) for side and front views, respectively]. Each of the three cells was filled with a mixture containing $0.25 \%(\mathrm{v} / \mathrm{v}) 0.1 \mu \mathrm{m}$ latex microspheres, and $0.64 \%(\mathrm{v} / \mathrm{v})$ agarose. Cells (i) and (ii) also contained $400 \mu \mathrm{M}$ ICG $\left(N_{0}=24 \times 10^{16} \mathrm{~cm}^{-3}\right)$ dissolved in $0.5 \% \mathrm{BSA}$ and DI water, respectively, whereas the third cell [marked (iii)] contained additional $0.125 \%(\mathrm{v} / \mathrm{v}) 1.437 \mu \mathrm{m}$ latex microspheres in order to bring the total attenuation of the cell contents close to that of the mixtures in (i) and (ii).

To acquire B-scans [two-dimensional (2D) images], the glass cuvette was mounted on a motorized linear stage in order to move the sample under the probe beam. The PFOA was programmed such that light exposure levels at the glass cuvette for OCT signal acquisition and ICG photobleaching were $60 \mu \mathrm{W}$ and $10 \mathrm{~mW}$, respectively. The linear detector array integration time per A-scan was set to $1 \mathrm{~ms}$. First, a B-scan image composed of $600 \mathrm{~A}$-scans was acquired with a $20 \mu \mathrm{m}$ lateral scan step [see Fig. 10(c)]. During the second B-scan, the sample was also photobleached using $\sim 19 \mathrm{~kW} / \mathrm{cm}^{2}$ irradiance at each A-scan position. Although the estimated time to photobleach the $400 \mu \mathrm{M}$ ICG samples was less than $0.5 \mathrm{~s}$, due to irradiance decay through the highly scattering phantoms (as noted earlier) we had to increase the exposure time for effective photobleaching. In summary, the phantoms were photobleached for $\Delta t=10 \mathrm{~s}$ duration at each A-scan location during the second B-scan, which is shown in Fig. 10(d). During both B-scans, an OCT sample size of 50 repeats was acquired at each A-scan position in order to reduce speckle. A comparison of Figs. 10(c) and 10(d) shows that cells marked as (i) and (ii) containing samples of ICG in $0.5 \%$ BSA and water, respectively, show significant increase in backscatter from the latex microspheres. This enhanced backscatter is attributable to change in absorption coefficients of the ICG samples in the first two cells. Figure 10(e) shows averaged A-scans [at the marked location in cell (ii)] before and after the photobleaching; the corresponding ratio of the averaged A-scans is shown in Fig. 10(g). Using Eq. (13), the average bleached state population density $N_{B}$ of ICG molecules within cell (ii) was determined as $\sim 23 \times 10^{16} \mathrm{~cm}^{-3}$, indicating that $\sim 95 \%$ ICG molecules were transitioned to the photobleached state. The third cell [marked (iii)] showed no change in backscatter [also see Figs. 10(f) and 10(h) for representative A-scans and their ratio at the marked location] indicating that regions without ICG do not yield any contrast in the proposed pump-probe scheme for MCOCT.

To demonstrate the ICG detection capability in an animal model, a mixture of $400 \mu \mathrm{M}$ ICG, $0.25 \%$ (v/v) $0.1 \mu \mathrm{m}$ latex microspheres, and $0.6 \%(\mathrm{v} / \mathrm{v})$ of agarose was used to fill the gill arch cavities of euthanized stage 54 Xenopus laevis [see Fig. 11(a)]. First, the PFOA was set to illuminate the sample with $60 \mu \mathrm{W}$ and a whole B-scan (400 A-scans; lateral resolution $\sim 7.5 \mu \mathrm{m}$ ) was acquired by moving the sample with a motorized linear stage [see Figs. 11(b) and 11(d)]. During the second B-scan, the PFOA was set to illuminate the sample with $\sim 10 \mathrm{~mW}$ power at each A-scan position for $12 \mathrm{~s}$ duration. The optical power was then readjusted to $60 \mu \mathrm{W}$ to acquire the corresponding A-scan, and so forth. During both B-scans, an OCT sample size of 50 repeats was acquired at each A-scan position in order to reduce speckle. Figures 11(c) and 11(e) show the second B-scan where increased backscatter from the microspheres (indicating the localization of ICG) in gill arch cavities can be clearly seen. This increased backscatter, attributable to the change in the absorption coefficient of ICG from within the gill arch cavities of the tadpole, demonstrates the functionality of ICG as a contrast agent in the proposed pump-probe scheme. Further analysis of the xenopus images to estimate achieved contrast could not be carried out due to sample shifting between the two B-scans (acquired before and after the photobleaching).

\section{Considerations of ICG-Based Pump-Probe Scheme for Clinical Applications}

This MCOCT scheme can be applied in animal model studies to tomographically visualize body cavities, blood vessel systems, and gastrointestinal tracts. The fact that ICG is approved for clinical usage points to possible usage of this MCOCT scheme for clinical applications as well. While the sensitivity achieved in our study is insufficiently high for clinical applications, the findings of our study do form a basis by which we can evaluate the improvements needed for this MCOCT scheme and other similar MCOCT schemes to be clinically applicable.

There are two major considerations that we need to account for. First, is it possible to deliver the fluence needed for photobleaching in a reasonable time frame? Second, is the method sufficiently sensitive?

The time frame for fluence delivery can be estimated by the following approach. The American National Standards Institute (ANSI) guidelines for skin exposure ${ }^{31}$ suggest that an acceptable light illumination for long time frame exposure is $300 \mathrm{~mW} / \mathrm{cm}^{2}$. For a nominal ICG concentration of $10 \mu \mathrm{M}$, the approved ICG concentration in blood for clinical procedures, ${ }^{32}$ photobleaching of $25 \%$ of the dye will require a time duration of $350 \mathrm{~s}$. This time frame can be reduced by using a higher irradiance or switching to a dye with a higher quantum yield for photobleaching. This estimate implies that using ICG with this MCOCT scheme is not well-suited for time critical clinical procedures.

The sensitivity of the method can be estimated by noting that the fundamental limit on the contrast agent sensitivity is related to the SNR of the underlying OCT system as ${ }^{22}$

$$
\begin{aligned}
S N R_{M C O C T}= & \left(\frac{\varepsilon P_{s} T}{h \nu}\right) \times R(z) \exp \left[-2 \int_{0}^{z} \mu_{a, s}\left(\lambda, z^{\prime}\right) d z^{\prime}\right] \\
& \times\left[\left|\Delta \mu_{a}(z)\right| \Delta L\right]^{2},
\end{aligned}
$$

where, $\varepsilon$ is the detection sensitivity, $P_{S}$ is the collected sample power, $T$ is integration time, and $h \nu$ is the energy of photons. $R(z)$ is the sample reflectivity at depth $z$, whereas $\mu_{a, s}$ is the overall absorption and scattering extinction coefficient. $\Delta \mu_{a}$ represents the change in absorption coefficient of the thin absorptive layer of thickness $\Delta L$ at depth $z$ inside the scattering sample. The fundamental limit on the contrast agent sensitivity can be determined by setting Eq. (14) equal to 1 . To determine the minimal concentration of ICG detectable by our MCOCT system, we use the measured OCT SNR of $98 \mathrm{~dB}$ at $1 \mathrm{~ms}$ integration time. By assuming a nominal $R(z)=10^{-4}$ (typical scattering from tissue), we find out that the minimal 
Yaqoob et al.: Molecular contrast optical coherence tomography...

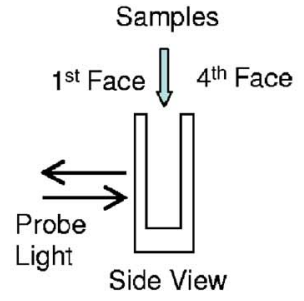

(a)

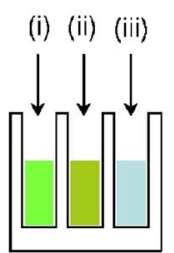

Front View

(b)

(c)

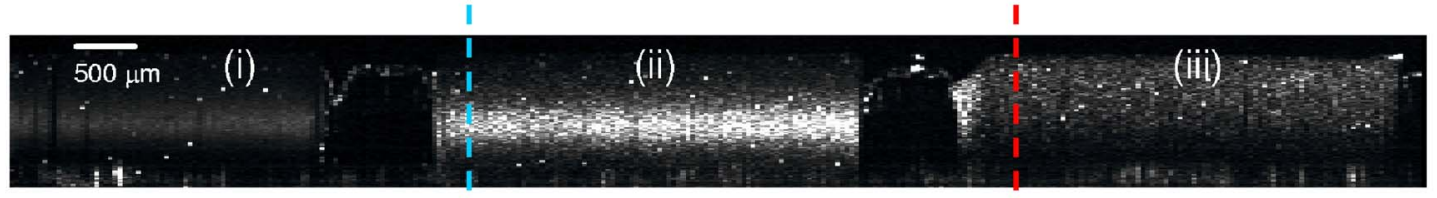

(d)

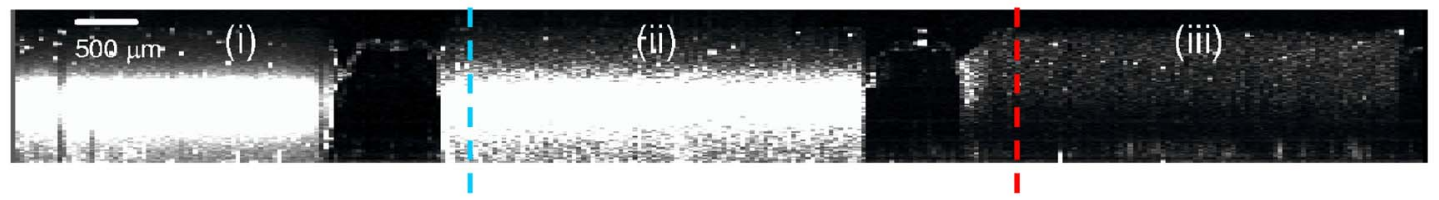

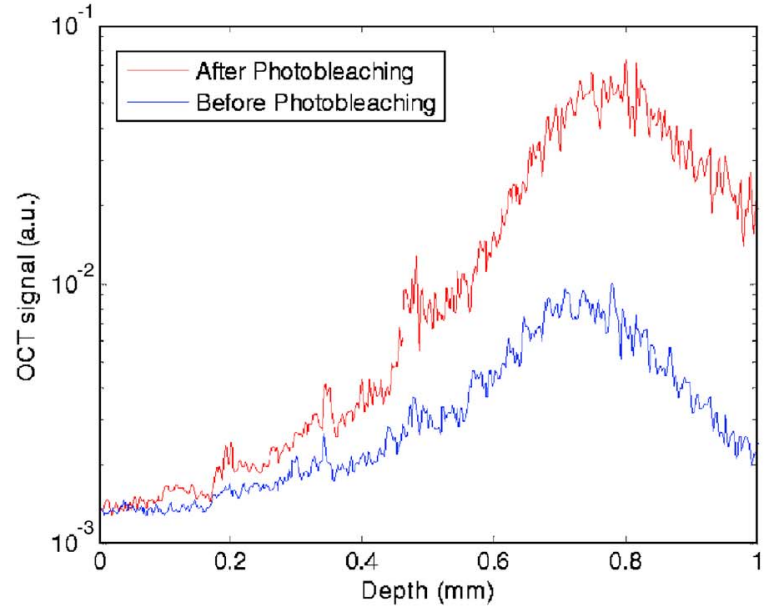

(e)

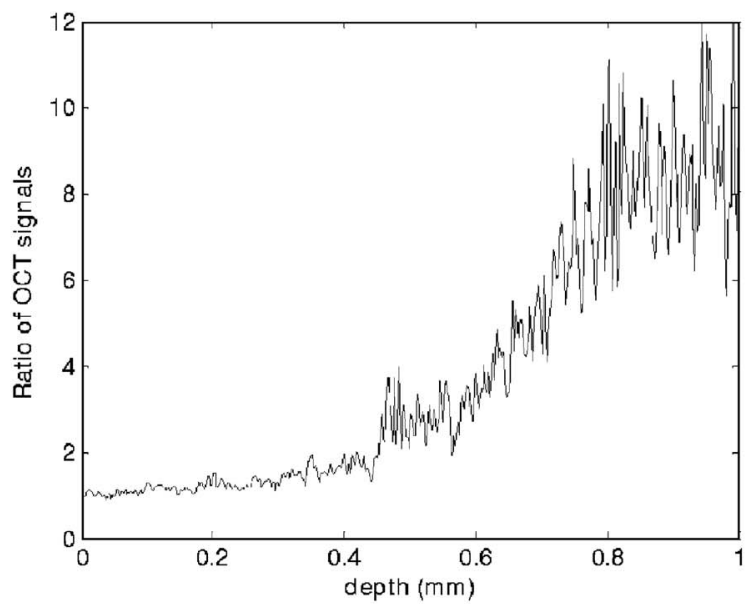

(g)

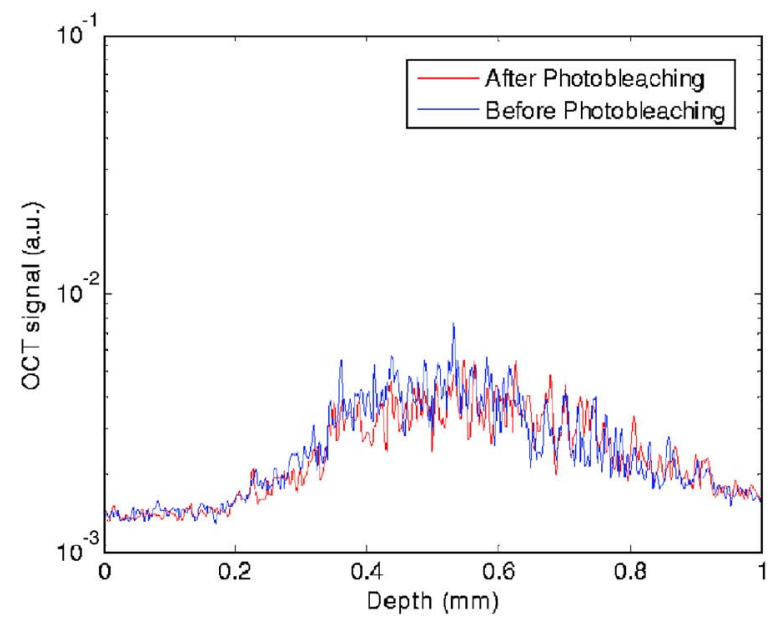

(f)

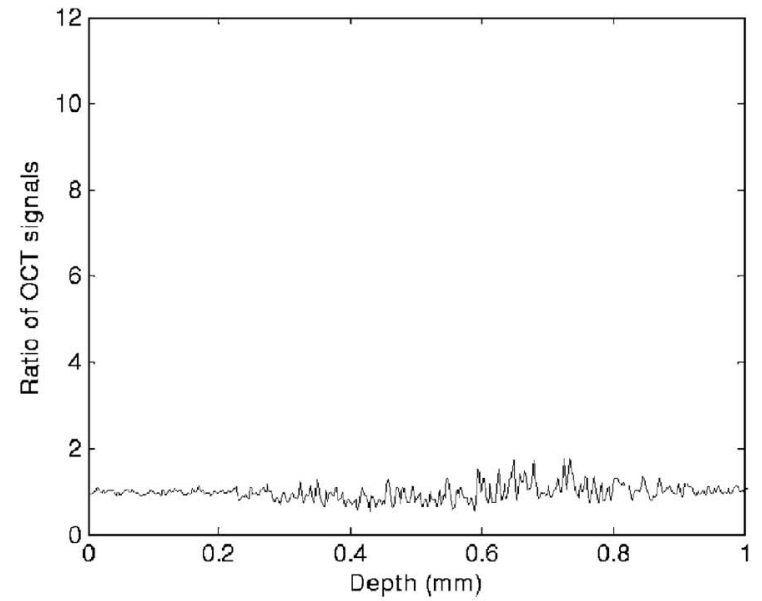

(h)

Fig. 10 (a) Side and (b) front view of a cuvette composed of 3 cells filled with a mixture containing $0.25 \%$ ( $/ \mathrm{v}$ ) $0.1 \mu \mathrm{m}$ latex microspheres and $0.64 \%(\mathrm{v} / \mathrm{v})$ agarose. Cells (i) and (ii) also contained $400 \mu \mathrm{M}$ ICG dissolved in 0.5\% BSA and DI water, respectively. Cell (iii) contained additional $0.125 \%(\mathrm{v} / \mathrm{v}) 1.437 \mu \mathrm{m}$ latex microspheres to bring the total attenuation of the cell contents close to that of the mixtures in (i) and (ii). (c) and (d) show B-scan images (600 A-scans each) of the glass cuvette, shown in (a), acquired with $20 \mu \mathrm{m}$ lateral scan step before and after ICG photobleaching, respectively. (e) and (g) show averaged A-scans of cells (ii) and (iii) before and after high intensity illumination, respectively, at the locations marked by dotted lines; the corresponding ratios of the averaged A-scans are shown in (f) and (h). 


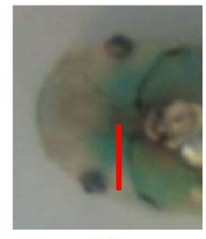

(a)

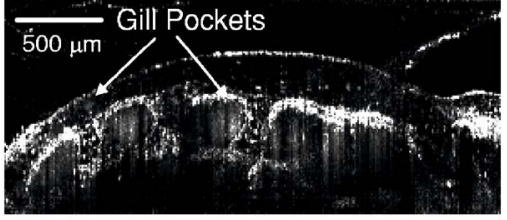

(b)

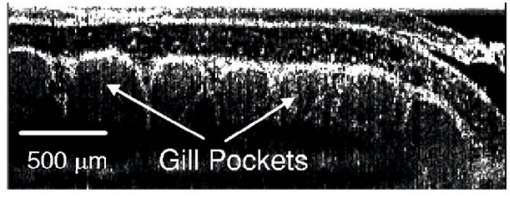

(d)

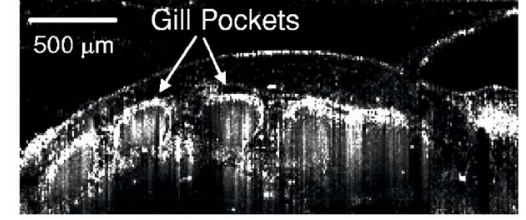

(c)

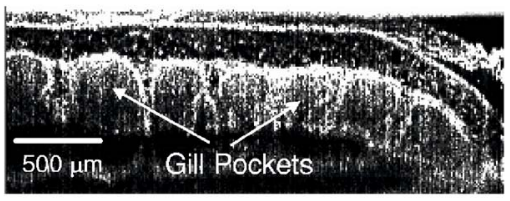

(e)

Fig. 11 (Color online only) MCOCT imaging of a stage 54 Xenopus laevis. (a) Ventral view of the animal filled with a mixture of $400 \mu \mathrm{M}$ ICG (green), $0.25 \% 0.1 \mu \mathrm{m}$ latex microspheres, and $0.6 \%$ volume concentration of agarose; the red line indicates the region where OCT scans were acquired. (b) and (c) show B-scan images of the illuminated region before and after ICG photobleaching, respectively. (d) and (e) show B-scan images of gill arch cavities of another stage 54 Xenopus laevis. Increased backscatter from within the gill arch cavities of the animal can be seen.

detectable concentration for $\Delta L=100 \mu \mathrm{m}$ thick ICG layer at $z=0$ is $0.8 \mu \mathrm{M}$. This concentration compares very favorably with the clinically safe ICG concentration of $10 \mu \mathrm{M}$ in blood. $^{32}$

Our experiments show that this sensitivity is not reached because the above analysis assumes that the OCT signal does not show significant variation from scan to scan. While this assumption is true for immobile targets, the presence of fluid and the associated speckle fluctuations lead to significant scan-to-scan signal variation. This variation can be as large as 4 to $5 \mathrm{~dB}$ from scan to scan in our experiments. While averaging can help reduce the variation, this variation limited our ability to discern contrast. The reduction of speckle fluctuations is crucial to enabling this and similar MCOCT schemes to reach the contrast sensitivity given in the above analysis.

\section{Conclusions}

In conclusion, we have proposed a pump-probe scheme for MCOCT imaging of biological samples using ICG as a contrast agent. The photobleached state in ICG is permanent in nature that makes this transition useful in the reported pumpprobe scheme. A photobleaching study of $50 \mu \mathrm{M}$ ICG solutions in DI water, BSA, and agarose shows that the cumulative probability of photobleaching decreases with fluence in all the cases. Various concentrations (ranging from 12 to $137 \mu \mathrm{M})$ of ICG in DI water were also studied, which illustrates that $\phi_{B \text {,cum,init }}$ decreases with the increasing dye concentration. We also found that at higher fluence levels, the final values of cumulative probability (for the range of ICG concentrations used in the study) tend to converge to $(2.7 \pm 0.6) \times 10^{-6}$. The final cumulative probability $\phi_{B, \text { cum,final }}$ is useful in estimating the extent of photobleaching in a given sample of ICG for known irradiance and exposure time. Using our laboratory spectral domain MCOCT setup, we have successfully mapped the distribution of ICG (400 $\mu \mathrm{M}$ concentration) within tissue phantoms as well as stage 54 Xenopus laevis. The ability to detect and map depth-resolved distribution of ICG within a biological sample illustrates the potential of the proposed pump-probe scheme to provide high resolution structural information of tissue as well as the ability to differentiate between certain tissue pathologies.

As opposed to spectral triangulation MCOCT, ${ }^{20}$ which is based on spectral differential absorption of ICG, the proposed pump-probe scheme relies upon the change in absorption spectrum of ICG due to photobleaching. Since the proposed method uses the permanently photobleached state in ICG (the photobleached dye molecules do not relax back to the ground state), both the optical design as well as the associated data acquisition methodology are simple.

However, the proposed technique does rely on its ability to effectively photobleach the dye. The current pump-probe MCOCT scheme is limited in imaging speed due to the low probability of photobleaching, which requires high irradiance and relatively long exposure time to photobleach ICG. For instance, the estimated light exposure level required for complete photobleaching of $10 \mu \mathrm{M}$ ICG is 4 to 5 orders of magnitude higher than the ANSI prescribed limits. Previously, it has been shown that the probability of ICG photobleaching decreases in the presence of certain materials such as silver particles. A study of materials that would potentially increase the probability of ICG photobleaching upon conjugation would benefit the proposed ICG-based pump-probe MCOCT scheme. Using higher irradiance (subject to the availability of high power light source) and modifying the system accord- 
ingly, so as to photobleach the entire region of interest as opposed to line-by-line photobleaching (the current methodology), we can improve the overall imaging speed of the system.

\section{Acknowledgment}

This research was supported by National Institute of Health Grant No. 1RZ1EB004602-1.

\section{References}

1. D. Huang, E. A. Swanson, C. P. Lin, J. S. Schuman, W. G. Stinson, W. Chang, M. R. Hee, T. Flotte, K. Gregory, C. A. Puliafito, and J. G. Fujimoto, "Optical coherence tomography," Science 254, 1178-1181 (1991).

2. B. Povazay, K. Bizheva, A. H. Unterhuber, B. Hermann, H. Sattmann, A. F. Fercher, W. Drexler, A. Apolonski, W. J. Wadsworth, J. C. Knight, P. S. J. Russell, M. Vetterlein, and E. Scherzer, "Submicrometer axial resolution optical coherence tomography," Opt. Lett. 27, 1800 (2002).

3. M. R. Hee, J. A. Izatt, E. A. Swanson, D. Huang, J. S. Schuman, C. P. Lin, C. A. Puliafito, and J. G. Fujimoto, "Optical coherence tomography of the human retina," Arch. Ophthalmol. (Chicago) 113, 325332 (1995).

4. J. J. Kaluzny, M. Wojtkowski, and A. Kowalczyk, "Imaging of the anterior segment of the eye by spectral optical coherence tomography," Opt. Appl. 32, 581-589 (2002).

5. P. Targowski, M. Wojtkowski, A. Kowalczyk, T. Bajraszewski, M. Szkulmowski, and W. Gorczynska, "Complex spectral OCT in human eye imaging in vivo," Opt. Commun. 229, 79-84 (2004).

6. A. Unterhuber, B. Povazay, B. Hermann, H. Sattmann, A. ChavezPirson, and W. Drexler, "In vivo retinal optical coherence tomography at $1040 \mathrm{~nm}$-enhanced penetration into the choroid," Opt. Express 13, 3252-3258 (2005).

7. A. M. Rollins, R. Ung-arunyawee, A. Chak, R. C. K. Wong, K. Kobayashi, M. V. Sivak, and J. A. Izatt, "Real-time in vivo imaging of human gastrointestinal ultrastructure by use of endoscopic optical coherence tomography with a novel efficient interferometer design," Opt. Lett. 24, 1358-1360 (1999).

8. T. Q. Xie, M. L. Zeidel, and Y. Pan, "Detection of tumorigenesis in urinary bladder with optical coherence tomography: Optical characterization of morphological changes," Opt. Express 10, 1431-1443 (2002)

9. G. Tearney and B. Bouma, "Atherosclerotic plaque characterization by spatial and temporal speckle pattern analysis," Opt. Lett. 27, 533535 (2002).

10. B. E. Bouma, G. J. Tearney, H. Yabushita, M. Shishkov, C. R. Kauffman, D. D. Gauthier, B. D. MacNeill, S. L. Houser, H. T. Aretz, E. F. Halpern, and I. K. Jang, "Evaluation of intracoronary stenting by intravascular optical coherence tomography," Heart 89, 317-320 (2003).

11. X. J. Wang, T. E. Milner, J. F. de Boer, Y. Zhang, D. H. Pashley, and J. S. Nelson, "Characterization of dentin and enamel by use of optical coherence tomography," Appl. Opt. 38, 2092-2096 (1999).

12. J. A. Izatt, M. D. Kulkami, S. Yazdanfar, J. K. Barton, and A. J. Welch, "In vivo bidirectional color Doppler flow imagingof picoliter blood volumes using optical coherence tomograghy," Opt. Lett. 22, 1439-1441 (1997).

13. J. F. deBoer, T. E. Milner, M. J. C. van Gemert, and J. S. Nelson, "Two-dimensional birefringence imaging in biological tissue by polarization-sensitive optical coherence tomography," Opt. Lett. 22,
934-936 (1997).

14. U. Morgner, W. Drexler, F. X. Kartner, X. D. Li, C. Pitris, E. P. Ippen, and J. G. Fujimoto, "Spectroscopic optical coherence tomography," Opt. Lett. 25, 111-113 (2000).

15. R. Leitgeb, M. Wojtkowski, A. Kowalczyk, C. K. Hitzenberger, M. Sticker, and A. F. Fercher, "Spectral measurement of absorption by spectroscopic frequency-domain optical coherence tomography," Opt. Lett. 25, 820-822 (2000).

16. K. D. Rao, M. A. Choma, S. Yazdanfar, A. M. Rollins, and J. A. Izatt, "Molecular contrast in optical coherence tomography by use of a pump-probe technique," Opt. Lett. 28, 340-342 (2003).

17. T. M. Lee, A. L. Oldenburg, S. Sitafalwalla, D. L. Marks, W. Luo, F. J. J. Toublan, K. S. Suslick, and S. A. Boppart, "Engineered microsphere contrast agents for optical coherence tomography," Opt. Lett. 28, 1546-1548 (2003)

18. C. Vinegoni, J. S. Bredfeldt, D. L. Marks, and S. A. Boppart, "Nonlinear optical contrast enhancement for optical coherence tomography," Opt. Express 12, 331-341 (2004).

19. Y. Jiang, I. Tomov, Y. M. Wang, and Z. P. Chen, "Second-harmonic optical coherence tomography," Opt. Lett. 29, 1090-1092 (2004).

20. C. Yang, L. E. Lamb, J. D. Simon, M. A. Choma, B. Applegate, and J. Izatt, "Spectral triangulation molecular contrast optical coherence tomography with indocyanine green as the contrast agent," Opt. Lett. 29, 2016 (2004).

21. C. H. Yang, L. E. L. McGuckin, J. D. Simon, M. A. Choma, B. E. Applegate, and J. A. Izatt, "Spectral triangulation molecular contrast optical coherence tomography with indocyanine green as the contrast agent," Opt. Lett. 29, 2016-2018 (2004).

22. C. H. Yang, "Molecular contrast optical coherence tomography: A review," Photochem. Photobiol. 81, 215-237 (2005).

23. A. L. Oldenburg, F. J. J. Toublan, K. S. Suslick, A. Wei, and S. A. Boppart, "Magnetomotive contrast for in vivo optical coherence tomography," Opt. Express 13, 6597-6614 (2005).

24. M. V. Sarunic, B. E. Applegate, and J. A. Izatt, "Spectral domain second-harmonic optical coherence tomography," Opt. Lett., 30, 2391-2393 (2005).

25. H. Nimura, "Infrared ray electronic endoscopy combined with indocyanine green injection for detection of sentinel nodes of patients with gastric cancer," Br. J. Surg. 91, 575-579 (2004).

26. F. C. Piccolino, C. M. Eandi, L. Ventre, R. C. R. De la Longrais, and F. M. Grignolo, "Photodynamic therapy for chronic central serous chorioretinopathy," Retina 23, 752-763 (2003).

27. H. Gratz, A. Penzkofer, C. Abels, R.-M. Szeimies, M. Landthaler, and W. Baumler, "Photo-isomerisation, triplet formation, and photodegradation dynamics of indocyanine green solutions," J. Photochem. Photobiol., A 128, 101-109 (1999).

28. W. Holzer, M. Mauerer, A. Penzkofer, R.-M. Szeimies, C. Abels, M. Landthaler, and W. Baumler, "Photostability and thermal stability of indocyanine green," J. Photochem. Photobiol., B 47, 155-164 (1998).

29. J. E. Lee, T. J. Yoon, B. S. Oum, J. S. Lee, and H. Y. Choi, "Toxicity of indocyanine green injected into the subretinal space-Subretinal toxicity of indocyanine green," Retina 23, 675-681 (2003).

30. M. Wojtkowski, V. J. Srinivasan, T. Ko, J. G. Fujimoto, A. Kowalczyk, and J. S. Duker, "Ultrahigh-resolution, high-speed, Fourier domain optical coherence tomography and methods for dispersion compensation," Opt. Express 12, 2404-2422 (2004).

31. American National Standards Institute, "Safe Use of Lasers," in ANSI Z 136, pp. 1-2000, Laser Institute of America, Orlando, FL (2000).

32. P. E. Stanga, J. I. Lim, and P. Hamilton, "Indocyanine green angiography in choriorefinal diseases: Indications and interpretation-An evidence-based update," Ophthalmology 110, 15-21 (2003). 